\title{
Testing the equivalence principle in space after the MICROSCOPE mission
}

\author{
Anna M. Nobili ${ }^{1,2}$ and Alberto Anselmi ${ }^{3}$ \\ ${ }^{1}$ Department of Physics "E. Fermi”, University of Pisa, Largo B. Pontecorvo 3, 56127 Pisa, Italy \\ ${ }^{2}$ INFN-Istituto Nazionale di Fisica Nucleare, Sezione di Pisa, Largo B. Pontecorvo 3, 56127 Pisa, Italy \\ ${ }^{3}$ Thales Alenia Space Italia, Strada Antica di Collegno 253, 10146 Torino, Italy
}

(Received 9 March 2018; revised manuscript received 26 June 2018; published 21 August 2018)

\begin{abstract}
Tests of the weak equivalence principle (WEP) can reveal a new, composition dependent, force of nature, or disprove many models of new physics. For the first time, such a test is being successfully carried out in space by the MICROSCOPE satellite. Early results show no violation of the WEP sourced by the Earth for $\mathrm{Pt}$ and Ti test masses with random errors (after $8.26 \mathrm{~d}$ of integration time) of about 1 part in $10^{14}$ and systematic errors of the same magnitude. This result improves by about 10 times over the best ground tests with rotating torsion balances despite 70 times less sensitivity to differential accelerations, thanks to the much stronger driving signal in orbit. The measurement is limited by thermal noise from internal damping in the gold wires used for electrical grounding, related to their fabrication and clamping. This noise was shown to decrease when the spacecraft was set to rotate faster than planned. The result will improve by the end of the mission, as thermal noise decreases with more data. Not so systematic errors. We investigate major nongravitational effects and find that MICROSCOPE's "zero-check" sensor, with test masses both made of $\mathrm{Pt}$, does not allow their separation from the signal. The early test reports an upper limit of systematic errors in the Pt-Ti sensor, which are not detected in the Pt-Pt one, hence would not be distinguished from a violation. Once all the integration time available is used to reduce random noise, there will be no time left to check systematics. MICROSCOPE demonstrates the huge potential of space for WEP tests of very high precision and indicates how to reach it. To realize the potential, a new experiment needs the spacecraft to be in rapid, stable rotation around the symmetry axis (by conservation of angular momentum), needs high quality state-of-the-art mechanical suspensions as in the most precise gravitational experiments on ground, and must allow multiple checks to discriminate a violation signal from systematic errors. The design of the "Galileo Galilei" (GG) experiment, aiming to test the WEP to 1 part in $10^{17}$ unites all the needed features, indicating that a quantum leap in space is possible provided the new experiment heeds the lessons of MICROSCOPE.
\end{abstract}

DOI: 10.1103/PhysRevD.98.042002

\section{INTRODUCTION}

The general theory of relativity (GR) stands on the fundamental assumption that in a gravitational field all bodies fall with the same acceleration regardless of their mass and composition, a "fact of nature" known as the universality of free fall (UFF) or the weak equivalence principle (WEP). The WEP is at the crossroads of the open problems of fundamental physics: the relation of quantum fields and gravitation, the nature of dark matter and dark energy, the absolute character of the fundamental constants of physics. Tests of the WEP provide severe constraints to "new physics" attempting to cross the gap between GR and the Standard Model of particle physics, or make sense of dark matter and dark energy.

The dimensionless Eötvös [1] parameter

$$
\eta_{\text {Eötvös }}=\frac{\Delta a}{a}
$$

quantifies the level of violation. $\Delta a$ is the differential acceleration measured between two test masses of different composition as they fall in the field of a source body with average acceleration $a$ (the so-called "driving signal").

A reliable measurement of $\eta_{\text {Eötvös }} \neq 0$ would amount to the discovery of a new long-range composition dependent force of nature and make a revolution in physics; the higher the precision of the test, the higher the chances to find new physics. Conversely, the more sensitive the test yielding $\eta_{\text {Eötvös }}=0$, the greater the fine-tuning required for many physical models and theories to survive.

The best experiments, carried out by the Eöt-Wash group with slowly rotating torsion balances (RTB), have established that there is no violation to about 1 part in $10^{13}[2,3]$. While some improvement is still possible, gaining orders of magnitude requires moving the experiment to a laboratory in space (see [4] and references therein). An experiment to test the WEP in orbit, named STEP, has been studied since the 1970s [5-7]; in the 1990s, following the interest raised 
by a reanalysis of the Eötvös experiment [8], ESA and NASA have investigated the mission in considerable detail with the goal of testing the equivalence principle to $10^{-17}$ [9].

For the first time, an equivalence principle test is carried out with test masses in low Earth orbit, weakly suspended inside the MICROSCOPE spacecraft aiming to reach $10^{-15}$ [10]. With the signal at a few $\mathrm{mHz}$, MICROSCOPE scientists report for a Pt-Ti composition dipole, a null result relative to the Earth with a random noise on the Eötvös parameter $\eta_{\oplus}$ of about $10^{-14}$ (after an integration time of $8.26 \mathrm{~d}$ ) and systematic errors at the same level [11].

In the field of the Earth MICROSCOPE's early result is a tenfold improvement over rotating torsion balances. The improvement occurs despite a sensitivity to differential accelerations about 70 times worse than RTB at a similar frequency. In favor of the space test is the much larger driving signal from Earth by almost 500 times at a low altitude as compared to RTB on ground $\left(\sim 8 \mathrm{~ms}^{-2}\right.$ versus $0.0169 \mathrm{~ms}^{-2}$ at most) [4]. Having RTB superseded mass dropping tests by several orders of magnitude, despite a driving signal almost 600 times weaker $\left(\lesssim 0.0169 \mathrm{~ms}^{-2}\right.$ versus $\left.9.8 \mathrm{~ms}^{-2}\right)$, the very large factor yet to gain in low Earth orbit and the success of MICROSCOPE strongly indicate that the next leaps in precision tests of the WEP shall occur in space.

There is no such gain over RTB if data are analyzed taking the Sun or the dark matter in our Galaxy, as source bodies of a possible WEP violation. In this case, the gravitational and inertial forces which are being compared are the gravitational attraction from the source body (either the Sun or dark matter at the center of the Galaxy) and the centrifugal force that keeps the test masses in orbit around them, and there is no larger driving signal in low Earth orbit. The best null results in the field of the Sun and of dark matter have been established by RTB at $\eta_{\odot}$ and $\eta_{\mathrm{DM}}$ of a few times $10^{-13}$ and a few times $10^{-5}$, respectively ([3], Table 3). With a sensitivity to differential accelerations worse than RTB, no improvement can come from MICROSCOPE.

Candidate dark matter particles are typically new particles, not included in the Standard Model of particle physics, which would generate a long-range compositiondependent scalar interaction, hence violate the WEP. RTB tests rule out such a new composition dependent interaction between dark matter and ordinary matter to a few parts in $10^{5}$ [3] stating that, to this level of precision, dark matter in our Galaxy interacts with ordinary matter via the gravitational interaction only. Our current understanding of the cosmos is based on the assumption that the required nonluminous dark matter interacts with ordinary matter only gravitationally and there is no new long-range interaction. Although this assumption is very often taken for granted, we should be reminded that it is only an assumption, and as such, it should be tested by the most sensitive possible experiments.

In MICROSCOPE, each test cylinder is actively controlled by electrostatic forces (electrostatic suspensions act as a negative spring; hence, uncontrolled cylinders would be unstable [12]), and the readout is capacitive too. Electric grounding is ensured by connecting each cylinder to the cage with a thin gold wire which should have only an ancillary role. Nonetheless, the measurement is limited by thermal noise due to losses in the wires related to their fabrication and clamping.

Thermal noise due to internal damping in the suspensions decreases with the frequency as $1 / \sqrt{\nu}[13,14]$. A signal of WEP violation with the Earth as source would be DC on ground and at orbital frequency in space. A way to increase this frequency is by rotating the sensor relative to the Earth, the faster the better.

In MICROSCOPE, each test cylinder is sensitive in 1D, along its symmetry axis. Hence, rotation relative to the Earth must occur around an axis perpendicular to the symmetry axis ([11], Fig. 1), which is the only stable axis against small perturbations. Therefore, the rotation was planned to be slow, below $5 \nu_{\text {orb }}$ [10], but it has been raised in order to reduce thermal noise since it turned out to be higher than expected. The result reported in [11] has been obtained at $\nu_{\text {spin }}=17.5 \nu_{\text {orb }} \simeq 2.94 \mathrm{mHz}$, and this is the current baseline. This is the first demonstration of a high precision rotating experiment in space. Rotation of the whole spacecraft relative to inertial space, with no stator and no bearings, has very low noise and is the key to the mission success.

By the end of the mission, with more data available, thermal noise, being random, will decrease and allow a WEP test closer to the $10^{-15}$ original target of the mission. The upper limit of currently reported systematic errors is about 10 times larger than that. They will not disappear or decrease with more data, and all systematics that eventually

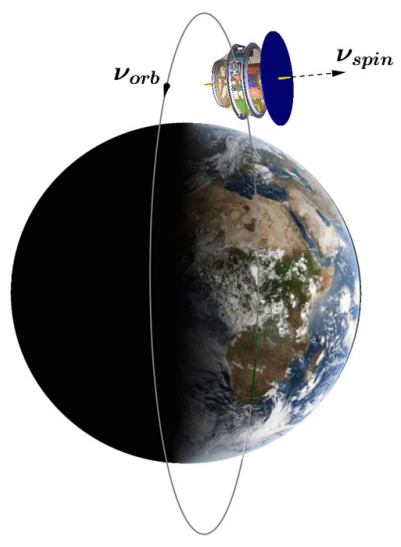

FIG. 1. Sketch of the GG satellite, with cylindrical symmetry and the dish of solar cells facing the Sun, in a high inclination sun-synchronous orbit $\left(\nu_{\text {orb }} \simeq 1.7 \times 10^{-4} \mathrm{~Hz}\right)$ similar to that of MICROSCOPE. The spacecraft is passively stabilized by oneaxis rotation around the symmetry axis at $\nu_{\text {spin }}=1 \mathrm{~Hz}$. After initial spin up relative to inertial space, rotation is maintained by conservation of angular momentum. (Figure not to scale; the bulk of the spacecraft body has a diameter of about $1.5 \mathrm{~m}$ ). 
were to emerge above random noise shall require very careful checking in order to be separated with certainty from a possible violation signal.

For this purpose, MICROSCOPE carries a second, "zero-check" equal composition accelerometer (named SUREF) with the test cylinders both made of Pt. Ideally, a violation signal should appear in the Pt-Ti sensor and not in the Pt-Pt one, while systematic effects due to known physics should be detected by both sensors. In this work, we compare random noise and nongravitational systematic effects in the two sensors showing that the expected separation of the violation signal from systematic errors does not occur.

A thorough check of systematic errors cannot be avoided. It requires a sufficient number of measurements, all to the same precision, to be carried out in different physical conditions such that the different physical parameters involved allow the signal to be distinguished from systematics on the basis of their different signature, hence of their different dependence on these parameters.

MICROSCOPE scientists planned to reach the mission target $\eta_{\oplus}=10^{-15}$ with an integration time corresponding to 120 orbits, so that in a 2-yr mission duration, there would be many such measurements, making it possible to check the result and possibly even improve it. The published data show, however, that in 120 orbits, the measurement is about a factor of 10 short of the target. If all the remaining integration time is used to reduce random noise, there will be none left to check systematics.

The only way to resolve the ambiguity between systematic errors and a possible violation signal is by flying another experiment with higher precision and a shorter integration time. If care is taken in flying an experiment somewhat more sensitive than ground balances, the improvement achievable in space can be impressive. Rapid rotation of the spacecraft and consequent upconversion of the signal to high frequency, at which thermal noise is low and integration time is short, are the main drivers of the "Galileo Galilei" (GG) space experiment to test the equivalence principle to $10^{-17}$ without invoking cryogenics ([4,14-17]).

The paper is organized as follows.

In Sec. II, we present the early MICROSCOPE results, compare them with RTB, show how faster rotation has reduced thermal noise, discuss the reported systematic errors and their current limited understanding.

In Sec. III, we quantitatively compare the effects of thermal noise from internal damping and of major systematic errors in the two sensors, to find that at a precision closer to the $10^{-15}$ mission target, the Pt-Pt sensor will not allow a violation signal to be separated out. With not enough time left to confirm or rule out a violation, another experiment in space is needed; in Sec. IV, we argue that MICROSCOPE itself, through its success and limitations, shows that a much more precise test of the WEP in orbit is possible and points out the key changes to be made in order to achieve it.

In Sec. V, we draw the conclusions.

\section{MICROSCOPE FIRST TEST OF THE EQUIVALENCE PRINCIPLE IN SPACE: SUCCESS, LIMITATIONS, AND OPEN ISSUES}

While MICROSCOPE is still in orbit, early results with an integration time of $8.26 \mathrm{~d}$ yield, for Earth as the source body and test masses made of $\mathrm{Pt}$ (with $10 \%$ of $\mathrm{Rh}$ ) and $\mathrm{Ti}$ (with $10 \%$ of $\mathrm{Al}$ ), a null result at $1 \sigma$ level of [11],

$$
\eta_{\oplus}(\mathrm{Pt}, \mathrm{Ti})=[-1 \pm 9(\text { stat }) \pm 9(\text { syst })] \times 10^{-15} .
$$

It has been obtained with the spacecraft in low Earth orbit at a frequency $\nu_{\text {orb }}=0.16818 \mathrm{mHz}\left(P_{\text {orb }}=5946 \mathrm{~s}\right)$ rotating at $\nu_{\text {spin }}=2.9432 \mathrm{mHz}\left(P_{\text {spin }}=339.8 \mathrm{~s}\right)$, whereby a WEP violation signal would occur at $\nu_{\mathrm{EP}}=\nu_{\text {spin }}+\nu_{\text {orb }}=$ $3.1113 \mathrm{mHz}\left(P_{\mathrm{EP}}=321.4 \mathrm{~s}\right)$.

By comparison with the best tests of WEP achieved on ground by RTB $[2,3]$, this is about a tenfold improvement. The improvement occurs with a sensitivity to differential accelerations at the signal frequency of

$$
\Delta a_{\mathrm{Pt}-\mathrm{Ti}} \simeq 9 \times 10^{-15} g(h) \simeq 7.1 \times 1 \mathrm{~ms}^{-2},
$$

where $g(h) \simeq 7.9 \mathrm{~ms}^{-2}$ is the average gravitational acceleration from Earth at $710 \mathrm{~km}$ altitude. Instead, RTB are sensitive to $\Delta a=10^{-15} \mathrm{~ms}^{-2}$ at a signal frequency of $0.84 \mathrm{mHz}$ for both the Be-Ti and Be-Al composition dipoles tested ([2,3], Table 3). Despite 70 times less sensitivity, MICROSCOPE's early test is about 10 times better thanks to the larger driving signal from Earth in orbit versus RTB on ground [4].

In low Earth orbit, air drag and solar radiation pressure acting on the outer surface of the spacecraft give rise to an equal and opposite inertial acceleration on every test mass weakly suspended inside the spacecraft many orders of magnitude smaller than 1-g, but also many orders of magnitude bigger than the target violation signal. The effect is common mode in principle, but in real experiments a differential residual remains much larger than the signal and at the same frequency. For MICROSCOPE, the inertial acceleration resulting from drag is roughly 7 orders of magnitude bigger than the signal.

At 1- $g$ the torsion balance can reach a relative precision of 1 part in $10^{13}$ in the differential effect of WEP violation having been built with tolerances of only 1 part in $10^{5}$, thanks to its capability to reject common mode effects. In MICROSCOPE, the test cylinders are suspended individually (they do not form a balance), and their configuration is frozen after assembling. Any difference resulting from construction and mounting errors can only be mitigated (rejected) by in-flight calibrations of their individual 
responses. Matching by $8.5 \times 10^{-3}$ (rejection factor of about 118) is reported in [11]. Such a low level of rejection means that in order to achieve the result (2) most of the drag acceleration has been successfully compensated by the drag-free control system of the spacecraft with propellant and thrusters. If combined with a comparable rejection level by means of an appropriate differential design of the sensor itself, it would allow a much higher precision to be reached without stringent requirements on thruster noise [18].

In MICROSCOPE, each test cylinder is weakly suspended along its symmetry axis by means of electrostatic forces, with a $600 \mu \mathrm{m}$ gap. Even after compensation of the large effect of drag, a restoring force is needed in response to small residual forces in order to prevent each cylinder from hitting the cage. Since electrostatic suspensions are unstable (they act as a "negative" spring [12]), a restoring force-including the response to the violation signal, if any-is provided by active electrostatic control.

In this type of accelerometers developed in France at ONERA, a loose, thin, conducting wire made of gold (7 $\mu \mathrm{m}$ width and $2.5 \mathrm{~cm}$ length in MICROSCOPE [19]) provides a physical connection between the test mass and the cage, primarily to avoid electric charging.

Concern about thermal noise from internal damping in the wire at the low frequencies of interest has led to extensive measurements of its quality factor $Q$ under realistic flight conditions. An ad hoc, electrostatically suspended torsion pendulum was built in order to avoid the suspension wire and thus, achieve a very weak torsional constant [20-22]. The electrostatic pendulum is in fact 10 times stiffer than the mechanical torsion pendulum of the Eöt-Wash group [2]. It has measured the $Q$ of the gold wire (7.5 $\mu \mathrm{m}$ width and $1.7 \mathrm{~cm}$ length in this case) at frequencies ranging from about $10^{-4} \mathrm{~Hz}$ to several $10^{-2} \mathrm{~Hz}$, showing the presence of large losses, with better $Q$ occurring at higher frequencies. The values measured range from $Q=36$ slightly above $10^{-4} \mathrm{~Hz}$ (the orbital frequency) to 59 at $10^{-3} \mathrm{~Hz}$, while $Q$ values around 110 are measured only at frequencies of $10^{-2} \mathrm{~Hz}$ and a few times $10^{-2} \mathrm{~Hz}$ ([21], Fig. 5).

These losses are much higher than in the suspensions of small force ground experiments. At a signal frequency slightly less than a $\mathrm{mHz}$, the Eöt-Wash group reports a quality factor 100 times better than measured by ONERA at $1 \mathrm{mHz}$, of about 6000 , with a $20 \mu \mathrm{m} \mathrm{W}$ wire suspending a 70 gram balance at $1-g[2,3]$. Large losses also at low frequencies can be avoided, especially for suspensions to be used in weightlessness conditions, where even large masses need very low stiffness. Monolithic suspensions, manufactured from a single block (to avoid relaxation of bending energy) with enlarged ends (to ensure that clamping is located far from where the flexure undergoes deformation during motion), and with appropriate heat treatments, are commonplace in small force gravitational experiments and have low losses. Instead, a gold wire clamped with droplets of glue at its ends, where most dissipation occurs, is bound to yield large losses. Moreover, losses will be unequal even if all wires are taken from the same coil and assumed to be perfectly identical, because the procedure used for clamping is hardly repeatable.

It has been known since 1990 [13] that thermal noise from internal damping in the suspensions of the test masses in gravitational wave detectors decreases with the frequency as $1 / \sqrt{\nu}$. This is how the Virgo/LIGO detectors around $100 \mathrm{~Hz}$ can be sensitive to displacements of the mechanically suspended mirrors as small as about $10^{-19} \mathrm{~m}$ [23]. A signal of WEP violation is at a much lower frequency: with Earth as the source body, it is DC on ground and at orbital frequency in space. For this reason, rotation of the apparatus is used, as for torsion balances, to up-convert the signal to higher frequencies, the higher the better.

Limitations to the spin rate of RTB come from concerns about rotation noise (on ground, it includes motor and bearings noise) and the attenuation of the signal strength at frequencies above the natural oscillation mode (the system being in essence a forced oscillator [14]). With a natural torsional frequency $\nu_{\text {tor }}=\frac{1}{798} \mathrm{~Hz}$, the highest spin rate so far is $\nu_{\text {spinRTB }}=\frac{2}{3} \nu_{\text {tor }} \simeq 0.84 \mathrm{mHz}$ [3].

In MICROSCOPE, rotation occurs perpendicularly to the orbit plane and to the symmetry axis ([11], Fig. 1), which however is the only stable axis against small perturbations. For this reason, a slow rotation mode was planned with $3 \nu_{\text {orb }}<\nu_{\text {spin }}<5 \nu_{\text {orb }}$ [10]. Once in orbit, thermal noise turned out to be higher than expected; being dominated by internal damping, the cure was to reduce it by increasing the rotation rate of the spacecraft. The result (2) was obtained while spinning at $\nu_{\text {spin }}=17.5 \nu_{\text {orb }} \simeq 2.94 \mathrm{mHz}$, which is 3.5 times faster than the maximum spin rate planned before launch. The possibility - unique to spaceof rotating the whole spacecraft, with no stator and no bearings, has proved to be much less noisy than rotating experiments in ground laboratories. The faster rotation rate has been adopted as the new baseline, despite the higher consumption of propellant and consequent shorter duration of the mission.

At a recent conference on "Fundamental Physics in Space" in Bremen, MICROSCOPE scientists have reported that when up-converting the signal frequency by increasing the rotation rate of the spacecraft, thermal noise in the Pt-Ti accelerometer improves more than expected according to the $1 / \sqrt{\nu}$ dependence [19]. The measurements show that while the signal frequency increases by a factor 3.53 , hence thermal noise from internal damping is expected to decrease by $\sqrt{3.53}=1.88$, it is instead found to decrease by 3.61 times, with an unexplained (favorable) factor of 1.9. The acceleration due to thermal noise from internal damping depends on the quality factor as $1 / \sqrt{Q}$ [see Eq. (4), Sec. III], and the quality factor too has been found to depend on the frequency, usually being higher (lower losses) at higher frequencies. If so, the unexplained 
improvement by a factor 1.9 might be due to an increase of the quality factor of the gold wires by $1.9^{2}=3.6$ times for the same system in the same conditions, except for the fact that the frequency of the signal has increased (with faster rotation) by 3.53 times. It is quite interesting that a similar improvement has been observed with the ground demonstrator of the proposed GG experiment in space: with a frequency increase by 2.16 times, the quality factor (for the same system, except for rotation) was found to increase by 2.24 times ([4], Sec. VIII).

The systematic errors shown in (2) have been found to be mostly of thermal origin. The electronics unit and the base plate of the Pt-Ti sensor were subjected to artificially produced thermal variations at the signal frequency, and the resulting differential acceleration between the two cylinders was measured, thus mapping the sensitivity of the instrument to thermal effects. Effects due to thermal variations of the base plate turned out to dominate over those of the electronics unit. But they were larger than expected by more than 2 orders of magnitude per degree of temperature variation, and the reasons for such behavior are not known yet. At the same time, by measuring the actual thermal variations (aside from those induced for the sensitivity test), it turned out that they were smaller than expected also by about 2 orders of magnitude [11]. Both findings call for a convincing physical explanation which may require more information, in particular on the residual pressure. MICROSCOPE scientists expect that thermal stability can be even better and therefore, consider the systematic error reported in (2) as an upper bound.

A rotation rate faster than planned may be responsible for a thermal stability better than expected, because of a better averaging and also because the signal frequency is farther away from the orbital frequency, at which most of the thermal stress obviously occurs.

\section{THERMAL NOISE, SYSTEMATIC ERRORS AND THE "ZERO-CHECK" SENSOR}

In addition to the composition dipole SUEP, whose inner (denser) cylinder is made of $\mathrm{Pt}$ (with $10 \%$ of $\mathrm{Rh}$ ) and the outer one of $\mathrm{Ti}$ (with $10 \%$ of $\mathrm{Al}$ ), MICROSCOPE carries a second sensor, named SUREF, with the same geometry (and the same $600 \mu \mathrm{m}$ gaps) as SUEP but cylinders made of the same material. The inner cylinder is "identical" to the inner one in SUEP, being made of the same Pt-Rh alloy (the two masses differ only by a few parts in $10^{4}$ ); the outer cylinder has the same size and volume as the outer one in SUEP, but it is made of Pt-Rh alloy like the inner one. All densities are uniform.

The Pt-Pt SUREF sensor should allow systematic errors to be distinguished from a violation signal ("zerocheck" sensor): a violation signal—being composition dependent - should appear in SUEP but not in SUREF, while systematic errors should appear in both sensors.
The two sensors are located $17.5 \mathrm{~cm}$ away from each other, and none of them is centered on the center of mass of the spacecraft. The drag-free control loop is closed either on one sensor or on the other, with the task of partially compensating the common mode motion, relative to the spacecraft, of the two selected cylinders due to the inertial acceleration (nominally the same for all test bodies) resulting from the effect of drag acting on the outer surface of the spacecraft. Depending on which sensor drives the drag-free control, science data are collected for that sensor only. Hence, SUEP and SUREF never take data simultaneously, which weakens the role of SUREF as a zero-check sensor and reduces the integration time available for SUEP to test the WEP.

MICROSCOPE scientists expect, by the end of the mission, to accumulate sufficient data for the random noise of SUEP shown in (2) to reduce to a value close to the $10^{-15}$ target of the mission. Then, unless systematic errors will turn out to be much lower than the upper limit currently established, they will emerge above noise and call for a full understanding of their physical origin, as they might contain a violation signal.

The Pt-Pt SUREF sensor should detect only systematic effects due to known physics, not a violation signal, and thus solve the problem. In reality, SUREF detection of systematic errors depends on its thermal noise (only those larger than thermal noise will be detected) and on its own sensitivity to systematics. If it turns out to be less sensitive than SUEP to some systematics, it cannot rule them out completely as a possible violation of the WEP.

The measurements are limited by thermal noise from internal damping in the gold wires. At the frequency of the violation signal $\nu_{\mathrm{EP}}$, the spectral density of the acceleration noise of each cylinder (expressed in $\mathrm{ms}^{-2} / \sqrt{\mathrm{Hz}}$ in SI units) reads [13]

$$
\widehat{a}_{\mathrm{thID}}=\frac{1}{\mathcal{M}} \sqrt{\frac{4 K_{B} T k_{w}}{Q_{w} \omega_{\mathrm{EP}}}},
$$

where $K_{B}$ is the Boltzmann constant, $T$ is the equilibrium temperature, $\mathcal{M}$ is the mass of the test cylinder, $k_{w}$ and $Q_{w}$ are the stiffness and quality factor of the gold wire connecting it to the cage, and $\omega_{\mathrm{EP}}=2 \pi \nu_{\mathrm{EP}}$ is the frequency of the signal. Being random noise, and most probably uncorrelated between the inner and outer cylinder in each sensor, the resulting differential acceleration noise competing with the signal is

$$
\widehat{\Delta a}_{\mathrm{thID}}=\sqrt{\widehat{a}_{\mathrm{thIDinner}}^{2}+\widehat{a}_{\mathrm{thIDouter}}^{2}} .
$$

Assuming the same ambient temperature $T$ in the two sensors and the same $k_{w}$ and $Q_{w}$ for all gold wires (even at different frequencies), the ratio of the differential acceleration noise between SUEP and SUREF is 


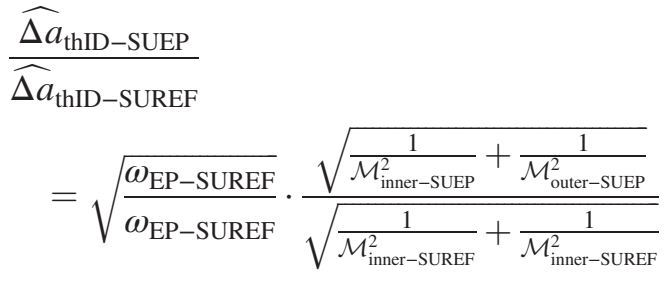

showing that it depends only on the different masses of the individual cylinders and on the different ratio between the frequencies of the signal, which in turn depends on the different rotation frequency during the respective measurements. The masses are measured very precisely, and their contribution to the noise ratio (6) is 1.5989 . We must therefore expect a higher thermal noise in SUEP than in SUREF by about 1.6, only because of the different values of the masses. The recent measurements for SUEP and SUREF have been made at different rotation rates of the spacecraft; hence, they refer to different signal frequencies whose ratio is $3.1113 \mathrm{mHz} / 0.9250 \mathrm{mHz}=3.3636$ [11], contributing to the noise ratio (6) by $1 / \sqrt{3.3636}=1 / 1.8340$. Overall, we get

$$
\frac{\widehat{\Delta a}_{\mathrm{thID}-\text { SUEP }}}{\widehat{\Delta a_{\mathrm{thID}-\text { SUREF }}}}=\frac{1.5989}{1.8340} \simeq 0.87,
$$

which means that at the selected rotation frequencies and with the assumptions made we should expect, in the differential acceleration noise competing with the signal, a slightly lower noise in SUEP than in SUREF. To the contrary, the measured values reported in [11] are $5.6 \times 10^{-11} \mathrm{~ms}^{-2} \sqrt{\mathrm{Hz}}$ for SUEP and $1.8 \times 10^{-11} \mathrm{~ms}^{-2} / \sqrt{\mathrm{Hz}}$ for SUREF, showing that SUEP is in fact 3.11 times more noisy. This means that there is an unexplained factor of about 3.57, SUEP being 3.57 times more noisy than SUREF than expected.

Since the temperature is well measured, only the differences in the ratio $k_{w} / Q_{w}$ for the test cylinders can be invoked, at least as long as random noise is due to internal damping as in (4). Since all four wires have the same length and are cut from the same coil, while $Q_{w}$ depends mostly on the glue clamping at the two ends of each wire-which are unpredictable and hardly repeatable- $Q_{w}$ is more likely to be responsible for the observed discrepancy. It appears in (4) under the square root; hence the $Q_{w}$, which dominates thermal noise in SUEP, should be almost $3.57^{2} \simeq 13$ times smaller than the one which dominates in SUREF.

Accelerometers similar in their key features to those of MICROSCOPE, also built by ONERA, have successfully flown onboard the GOCE geodesy mission of the European Space Agency, and a noise level about 2 times larger than expected has been reported in that case [24-26].

Concerning systematic effects at the frequency of the violation signal, and the respective sensitivities of SUEP and SUREF, we notice the following. The systematic errors which limit the Eötvös parameter (2) as measured with SUEP are depicted in [11], Fig. 3 left plot (and listed in Table III of the paper) at the level of about $7 \times 10^{-14} \mathrm{~ms}^{-2}$ as a function of time with the number $N$ of orbits (120 in total). The same plot shows the random acceleration noise, which instead decreases as $1 / \sqrt{N}$ to meet, towards the end of the run, the horizontal line of systematic errors. The same Fig. 3 (right plot) shows the (lower) random noise in SUREF, also decreasing as $1 / \sqrt{N}$ over a total of 62 orbits, to reach slightly below $3 \times 10^{-14} \mathrm{~ms}^{-2}$ at the end of the run. Should there be systematic effects as large as the upper limit reported in SUEP, they would clearly appear above random noise in SUREF, but no such systematics are detected. They do not appear either in the spectral density of the acceleration noise of SUREF shown in [11], Fig. 2 right plot. The question as to why it is so is obviously a very relevant one, because systematic errors should be detected by both SUEP and SUREF in order to be distinguished from a violation signal.

The systematic errors reported in SUEP and not detected in SUREF are nongravitational. We therefore compare the sensitivity of SUEP and SUREF to nongravitational perturbations. As shown in (1), in WEP tests, the physical observable is the differential acceleration of the test masses relative to the source body; hence, the relevant quantities are accelerations, not forces. The accelerations of a number of nongravitational perturbations are known to be proportional to the area-to-mass ratio of the affected body [27], the area being in this case that of the cross section of the test cylinder perpendicular to its sensitive/symmetry axis. The differential acceleration between the test cylinders in SUEP caused by such nongravitational perturbation would be

$$
\begin{aligned}
\Delta a_{n g \mathcal{A} / \mathcal{M}-\text { SUEP }} & =a_{n g \mathcal{A} / \mathcal{M} \text { outer-SUEP }}-a_{n g \mathcal{A} / \mathcal{M} \text { inner-SUEP }} \\
& \propto(\mathcal{A} / \mathcal{M})_{\text {outerSUEP }}-(\mathcal{A} / \mathcal{M})_{\text {innerSUEP }},
\end{aligned}
$$

where $(\mathcal{A} / \mathcal{M})$ is the area-to-mass ratio for the test cylinder referred to in the subscript; and similarly, for SUREF. If the physical parameters which determine the nongravitational perturbation under consideration are the same in both sensors, the ratio of the differential accelerations it gives rise to depends only on the ratios of $(\mathcal{A} / \mathcal{M})$ for the test cylinders in the two sensors

$$
\begin{aligned}
\frac{\Delta a_{n g \mathcal{A} / \mathcal{M}-S U E P}}{\Delta a_{n g \mathcal{A} / \mathcal{M}-\text { SUREF }}} \\
=\frac{(\mathcal{A} / \mathcal{M})_{\text {innerSUEP }}}{(\mathcal{A} / \mathcal{M})_{\text {innerSUREF }}} \\
\quad \frac{(\mathcal{A} / \mathcal{M})_{\text {outerSUEP }} /(\mathcal{A} / \mathcal{M})_{\text {innerSUEP }}-1}{(\mathcal{A} / \mathcal{M})_{\text {outerSUREF }} /(\mathcal{A} / \mathcal{M})_{\text {innerSUREF }}-1}
\end{aligned}
$$

With data available on the masses and the geometry of the test cylinders $[11,28,29]$, we get for this ratio (in modulus) 
about 3.3. Thus, nongravitational perturbations whose accelerations are proportional to the area-to-mass ratio of the test cylinders, give rise to differential accelerations 3.3 times larger in SUEP than in SUREF, simply because of the way they have been designed, all other physical parameters being the same.

Since the upper limit of systematic effects reported in SUEP at the frequency of the signal is of $7 \times 10^{-14} \mathrm{~ms}^{-2}$, we should expect systematics in SUREF to be a factor 3.3 smaller, hence of about $2 \times 10^{-14} \mathrm{~ms}^{-2}$ at most. Being below the thermal noise measured in SUREF ([11], Fig. 3 right plot), it is not surprising that they are not detected.

This fact questions the use of the zero-check Pt-Pt SUREF sensor to discriminate a violation signal from spurious effects. With a longer integration time and a lower thermal noise, SUREF may allow these systematics to be detected. However, as long as their value in SUEP is several times larger than in SUREF, the open issue remains that they may contain a violation signal as well.

Other nongravitational effects, such as electric charging, do not depend on the cross section but only on the inverse of the mass. In this case, the ratio of the differential accelerations between the test cylinders in the two sensors (all other physical parameters being the same) reads

$$
\begin{aligned}
\frac{\Delta a_{n g \mathcal{M}-S U E P}}{\Delta a_{n g \mathcal{M}-\text { SUREF }}}= & \frac{(\mathcal{M})_{\text {innerSUREF }}}{(\mathcal{M})_{\text {innerSUEP }}} \\
& \cdot \frac{(\mathcal{M})_{\text {innerSUEP }} /(\mathcal{M})_{\text {outerSUEP }}-1}{(\mathcal{M})_{\text {innerSUREF }} /(\mathcal{M})_{\text {outerSUREF }}-1},
\end{aligned}
$$

which yields 0.47 (modulus), meaning that this kind of systematic errors would be about 2 times larger in SUREF than in SUEP. Hence, electric charge effects, if any, are below the level of thermal noise reported in SUREF. Were such a spurious effect, at some point, detected above thermal noise in SUEP, the ratio (10) in favor of SUREF would not prevent its separation from the signal unless the observed ratio between the two effects is as theoretically expected.

Another disturbance competing with the signal, that questions the use of SUREF as a zero-check sensor is the radiometer effect, which is proportional to the residual pressure around the test cylinders and to the temperature gradient between the two ends of its sensitive/symmetry axis [30].

MICROSCOPE scientists exclude radiometer as the origin of the systematic effect in SUEP because of the extremely good thermal stability and uniformity observed. A residual pressure of $10^{-5} \mathrm{~Pa}$ was assumed before launch [10] but no value is given once in orbit. By comparison, LISA Pathfinder (LPF) established an upper limit of $2.2 \times 10^{-5} \mathrm{~Pa}$ at the start of the data take [31] and a few $10^{-6} \mathrm{~Pa}$ at the end of the experiment, because of venting to outer space and more time available for degassing [32].
There is no venting to outer space in MICROSCOPE, and the getter pumps it relies upon after final assembling in order to take care of pressure increase due to outgassing surfaces have a limited lifetime and are inefficient with noble gases. Thus, a reliable estimate of the residual pressure both in SUEP and in SUREF is needed, especially because different values may be expected due to the fact that the outer cylinder in SUEP is the only coated one of the four, and this may result in a different outgassing as compared to the outer cylinder in SUREF, despite the same geometry.

The radiometer effect should be carefully investigated because it is known to give no differential acceleration if the two cylinders have the same density, as in the case of SUREF [33]. We have checked this fact with the numbers available for the test cylinders [11,28], and the result for the ratio of the radiometer acceleration on the outer and inner cylinder in SUREF (of length $L_{\text {outer }}$ and $L_{\text {inner }}$, as in SUEP) is almost exactly 1 ,

$\frac{a_{\text {rad-outerSUREF }}}{a_{\text {rad-innerSUREF }}}=\frac{(\mathcal{A} / \mathcal{M})_{\text {outerSUREF }} \cdot L_{\text {outer }}}{(\mathcal{A} / \mathcal{M})_{\text {innerSUREF }} \cdot L_{\text {inner }}}=1.009$,

while the same ratio for SUEP is 4.562. Thus, the radiometer effect gives rise to a nonzero differential acceleration in SUEP and no differential acceleration in SUREF, just as one expects for the violation signal. As suggested in [33], a way out of this impasse might have been to fabricate the Pt-Pt cylinders in SUREF with a different average density, e.g., with some appropriate empty volume in one of them, e.g., the outer one.

Although some additional information may be available (by investigating the accelerations as measured individually by each test cylinder) that could help to mitigate the difficulties outlined here, it is likely that the main goal of the second equal composition sensor to provide a clearcut, unquestionable, check of the violation signal versus systematic errors will not be met.

If so, the need shall arise for carefully designed checks of systematic errors in the different composition sensor. This requires many measurements, all at the same sensitivity (possibly the target sensitivity) in different physical conditions, such that systematic errors and the violation signal can be distinguished on the basis of their respective signature and consequent different dependence on the physical parameters involved in these measurements, as it is done in ground tests of the WEP with RTB.

The additional complexity and cost of carrying a second sensor should better be used for flying two different composition dipoles instead of one. As argued in [2,3], their measurements can both be analyzed not only in the field of the Earth but also of the Sun and of dark matter at the center of our Galaxy, thus avoiding an accidental cancellation of the charges of the test-body dipole or the 
attractor. This would increase the chance of finding a nonnull result and strengthen its physical significance.

\section{LESSONS FROM MICROSCOPE AND ROOM FOR MAJOR IMPROVEMENTS}

With the zero-check sensor probably unable to firmly discriminate systematic errors, the final MICROSCOPE test of the WEP will have to rely on standard procedures of systematic error checks in the different composition sensor, as envisaged before launch [10] when the plan was to reach the mission target $\eta_{\oplus}=10^{-15}$ with an integration time corresponding to 120 orbits. In a 2-yr mission duration, there would be many such measurements to check the result and even improve it. In the words of MICROSCOPE scientists [10]: "The adopted trade-off remains on different sessions of 120 orbital periods. This is long enough to obtain the Eötvös parameter target exactitude of $10^{-15}$ in inertial mode and even better in rotating mode, by reducing the stochastic error with respect to the systematic evaluated one. This is also short enough to have time for many sessions with different experimental conditions."

It turns out that even in rotation mode, and at a faster rate than the maximum planned, the level of noise over 120 orbits is about 10 times higher than expected, and the entire mission duration is necessary to reduce it and bring the precision of the WEP test closer to the original $10^{-15}$ target. Since systematic errors are not expected to disappear with more data, and all the integration time available is used to reduce random noise, there will be no time left to check them.

We may therefore be left with the most sensitive test of the WEP ever, but no firm conclusion as to whether the equivalence principle is violated or not. Only another experiment in space, with a higher precision, shorter integration time and consequent reliable systematic checks, could give the answer. The success of MICROSCOPE, together with its limiting factors, tell us that in orbit it is possible to reach a much higher precision and give clear indications as to how to proceed in order to reach it.

High precision requires low thermal noise, which results in a short integration time. This needs a high $Q$ and high frequency of the signal to be obtained by rotating the spacecraft, the faster the better. As discussed in Sec. II, quality factors better than those of the gold wires of MICROSCOPE have been obtained, also at $\mathrm{mHz}$ frequencies. However, this improvement alone would not be enough to reach a sensitivity to differential accelerations better than that achieved by RTB.

A solution often advocated for MICROSCOPE is to eliminate any physical connection by replacing the gold wires with an active system of electric discharging, as in LPF. It would increase complexity and cost, but it is feasible. Once thermal noise from internal damping were eliminated by eliminating the suspensions, the next relevant one would be thermal noise from gas damping [34,35]. For the lowest possible residual pressure, a way to reduce gas damping noise is by increasing the gap, up to $4 \mathrm{~mm}$ in the case of LPF, as compared with $600 \mu \mathrm{m}$ in MICROSCOPE.

Using the actual data for the test cylinders and the gaps, and assuming a residual pressure of $10^{-5} \mathrm{~Pa}$ as planned before launch, we have calculated the differential acceleration noise due to gas damping in the SUEP sensor as it was done for GG [35]. The result is about $2 \times 10^{-13} \mathrm{~ms}^{-2} / \sqrt{\mathrm{Hz}}$, while for GG it was 2 orders of magnitude smaller, of about $2.8 \times 10^{-15} \mathrm{~ms}^{-2} / \sqrt{\mathrm{Hz}}$, thanks to the larger gaps $(2 \mathrm{~cm}$ vs $600 \mu \mathrm{m})$ and to the higher mass of the test cylinders (10 kg each vs 0.3 and $0.4 \mathrm{~kg}$ in SUEP). The value calculated for SUEP is 1 order of magnitude smaller than thermal noise from internal damping in the gold wires estimated before launch [10], and 2 orders of magnitude lower than the residual noise reported in orbit [11].

The capacitance is inversely proportional to the gap, so larger gaps mean a less precise readout, which in fact in LPF is at the nanometer level [36], while MICROSCOPE reports $3 \times 10^{-11} \mathrm{~m} / \sqrt{\mathrm{Hz}}$ between $2 \times 10^{-4} \mathrm{~Hz}$ and $1 \mathrm{~Hz}$ [11]. For LPF, this is not an issue because its main readout is based on laser interferometry [31]. Instead, MICROSCOPE relies on the capacitance readout; moreover, the capacitors control the test cylinders, which would otherwise be unstable. Note that gaps are already a factor of 2 larger than they were in GOCE, and a further increase is unlikely to yield a better net performance.

Thus, eliminating the gold wires is not going to improve the MICROSCOPE experiment; and the problem does not lie with the mechanical suspensions per se, as the most precise and most successful gravitational experiments (RTB and gravitational wave detectors) demonstrate. Mechanical suspensions at zero-g are perfectly predictable from ground measurements, and there is nothing mysterious about using them in space (see [37]). The stiffness can be predicted by semianalytical, seminumerical methods and verified experimentally in the required conditions of pressure and thermal stability and for the expected largest oscillation amplitude, by setting the flexure in oscillation in the horizontal plane so as not to be affected by local gravity. The quality factor at the frequency of interest is hard to predict, but it can be measured very reliably. There is certainly no need to fly a spring in order to establish its stiffness and quality factor.

Unlike electrostatic suspensions, mechanical suspensions act as positive springs and naturally provide the restoring force needed by the test masses. For instance, the deflection of the torsion balance under the effect of a torque with a nonzero component along the suspension fiber, including that of a WEP violation, is counteracted by its torsional elastic constant. In MICROSCOPE, the restoring force must be provided actively, while the gold wire acts as an ancillary dummy spring with the sole purpose of 
ensuring electric grounding. Instead, mechanical suspensions can provide both the restoring force and electric grounding. The torsion balance of the Eöt-Wash WEP experiments weighs only 70 grams in total and can be suspended with a very thin $\mathrm{W}$ wire of $20 \mu \mathrm{m}$ diameter whose torsional elastic constant is very low (being inversely proportional to the 4 th power of the thickness). In gravitational wave detectors, the mirrors to be suspended are much heavier (about $40 \mathrm{~kg}$ ) and the fibers much thicker (about $350 \mu \mathrm{m}$ ), but at frequencies around $100 \mathrm{~Hz}$ thermal noise from internal damping is very low. Suspension fibers are metallic in Virgo, soon to be replaced with fibers in fused silica (as in LIGO) with an even better quality factor. In orbit, weight is no longer a limitation, and large masses can be used, which reduces the effects of nongravitational forces, including those due to thermal noise. Thus, in space tests of the WEP, mechanical suspensions are preferable.

The solution we are led to is twofold. In the first place, we must use mechanical suspensions with state-of-the-art fabrication, heat treatment, and clamping procedures, so as to ensure high $Q$. Secondly, we must rotate the spacecraft much faster than MICROSCOPE, so as to up-convert the signal to a much higher frequency, where thermal noise from internal damping is significantly reduced.

MICROSCOPE has demonstrated the advantage of space for high precision rotating experiments. The possibility, unique to space, to spin the entire "laboratory", that is the spacecraft, along with the test cylinders makes rotation noise much lower in orbit than on ground. However, the spin rate of MICROSCOPE is limited by the need to rotate around an axis which is not the symmetry axis of the test cylinders ([11], Fig. 1). So far the highest reported spin rate, achieved during a SUEP 120-orbit run which has given the result (2), has been of $2.94 \times 10^{-3} \mathrm{~Hz}$. Spacecraft can spin much faster than that and be passively stabilized by rotation around the symmetry axis.

Mechanical suspensions are very versatile and allow the concentric test cylinders to be arranged in such a way that they corotate with the spacecraft around the symmetry axis, being sensitive in the plane perpendicular to it. In this plane, the relative displacements caused by tiny low frequency differential accelerations between the test cylinders - such as a violation signal—can be detected, upconverted by rotation to a much higher frequency where thermal noise is much lower [14]. After initial spin up, spacecraft stabilization is maintained passively by conservation of angular momentum, which ensures extremely low rotation noise and does not need propellant - to be left for drag-free control and occasional manoeuvres [17]. At Hz rather than $\mathrm{mHz}$ frequency, the thermal noise from internal damping is very low, and gaps can be increased so as to reduce also gas damping noise and make the integration time short even for a very high precision target [35]. With very low thermal noise, a readout of comparable low noise is needed. With $\mathrm{cm}$ level gaps a capacitance readout is not sensitive enough; a laser gauge with very low noise at $1 \mathrm{~Hz}$ is well feasible $[38,39]$.

The proposed GG space experiment incorporates all the features suggested by the MICROSCOPE experience and aims to test the WEP to 1 part in $10^{17}$ ([4,14-17]).

Like MICROSCOPE, it will fly in a low altitude, high inclination, sun-synchronous orbit (with orbital frequency $\nu_{\text {orb }} \simeq 1.7 \times 10^{-4} \mathrm{~Hz}$ ). However, thanks to its cylindrical symmetry (built around the concentric test cylinders), it can be passively stabilized by a one-axis rotation around the symmetry axis at the rotation rate $\nu_{\text {spin }}=1 \mathrm{~Hz}$ (Fig. 1 ). The coaxial, concentric test cylinders are located at the center of mass of the spacecraft, corotate with it, and are sensitive to differential forces acting in the plane perpendicular to the spin/symmetry axis. The relative displacements caused by any such force, like a violation signal, are read by a laser interferometry gauge, also corotating with the whole system. In the nonrotating frame of the spacecraft, the violation signal is at the orbital frequency; the laser gauge rotating at $\nu_{\text {spin }} \gg \nu_{\text {orb }}$ reads it at $\nu_{\text {spin }}$, where thermal noise from internal damping is much lower than it would be at $\nu_{\text {orb }}$, making the integration time much shorter [14,35]. This is the key to reaching a very high precision. It suffices to notice that improving by a factor of 10 in sensitivity requires - with a given level of thermal noisean integration time 100 times longer. As an example, the MICROSCOPE early test (2) has required 8.26 days of integration time; were it aiming at $10^{-17}$, the same experiment would need about 6.7 million days for one single measurement.

The expectations for GG, based on theoretical analysis, numerical simulations, and laboratory tests, are for a signalto-noise ratio of 2 in a few hours [35]. This allows a WEP test to $10^{-17}$ to be completed in $1 \mathrm{~d}$ (about 15 orbits). Then, since the spin axis (and the sensitive plane perpendicular to it) are fixed in inertial space, while the nodal line of the sunsynchronous orbit moves by about $1 \% \mathrm{~d}$ (for the solar panel to follow the annual motion of the Sun), a large number of 1-d runs shall be available, in different physical conditions provided by the dynamical evolution, to allow a violation signal at $10^{-17}$ level to be separated with certainty from systematic errors $[40,41]$.

As discussed in Sec. II, for a test of the WEP in orbit to reach a very high precision, it must deal with the huge effect of drag which requires, in addition to partial compensation by drag-free control, also a high level of common mode rejection (CMR). The largest common mode effect in orbit is the inertial acceleration equal and opposite to the acceleration of the spacecraft caused by nongravitational forces such as air drag and solar radiation pressure. A high level of CMR can be achieved if the test masses are arranged as a balance. This is how torsion balances have defeated mass dropping tests by many orders of magnitude. A torsion balance as such is not suitable for space [4]. However, space is favorable for the realization of 
a very sensitive balance because in orbit the largest common mode force against which the balance is balanced is many orders of magnitude weaker than 1-g on ground (a space version of the Watt balance has been considered at PTB, the German national metrology institute [42]).

In order to achieve a high level of CMR, the coaxial test cylinders in GG are arranged to form a beam balance, with the beam along the spin/symmetry axis, hence sensitive to differential forces in the plane perpendicular to it. The peculiarity of the GG balance is that, unlike ordinary beam balances, the two masses are concentric, which is a crucial requirement in space tests of the WEP to minimize classical differential tidal effects. The way such a beam balance with concentric test masses can be realized is based on an ingenious combination (originally designed by $\mathrm{D}$. Bramanti) of weak, high $Q$ flexures made in $\mathrm{CuBe}$ and coupling arms (with special attention to symmetry considerations) whose lengths can be finely adjusted to reach a very good balancing against the common mode inertial acceleration caused by drag. An animation of this balance is available on the front page of the GG website [16]. A 1-g version of it has been realized and tested in the lab with the "GG on ground" (GGG) demonstrator [4,15]. A second concentric sensor can be accommodated inside the GG spacecraft, whose test cylinders are arranged as a similar balance and can take data simultaneously with the first one [43]. As argued in Sec. III, it should better be a second composition dipole than an equal composition sensor.

GG will check MICROSCOPE's final result with at least 2 orders of magnitude better precision and improve by 4 orders of magnitude over RTB tests, thanks to the stronger signal in orbit (by about 500 times) and to a lesser extent by fully exploiting the advantages of space in order to reach a sensitivity to differential accelerations better than RTB (by 20 times). The latter factor would be an improvement also of RTB tests of the WEP relative to the Sun and to dark matter in our Galaxy.

\section{CONCLUSIONS}

MICROSCOPE demonstrates that, by going to space, a WEP experiment is within reach that can truly address the foundations of physics. For this potential to become real, numerous lessons from MICROSCOPE must be learned.

In this paper, we have shown that MICROSCOPE is limited by thermal noise due to the low quality factor of the gold wires used for electrical grounding, and yet outstanding results were achieved by rotating the spacecraft faster than planned. This fact establishes spacecraft rotation as the most effective way of improving the precision of WEP tests. Further, we have shown that the Pt-Pt "zerocheck" sensor is less sensitive to a wide class of systematic effects than the Pt-Ti sensor and therefore, can neither confirm nor disprove a violation signal at the achieved sensitivity level. Thus, there is no alternative to a rigorous campaign of systematic checks made up of many measurements in different experimental conditions, requiring short integration time, hence low thermal noise.

All these facts point the way to the design required of a breakthrough experiment. A new experiment must feature high quality, state-of-the-art mechanical suspensions as demonstrated in torsion balance ground tests of the WEP and in gravitational wave detectors. For low thermal noise, it must up-convert the signal to a much higher frequency, which is easily achieved in a fast rotating spacecraft, provided its cylindrical symmetry replicates that of the test bodies in a Russian doll setup with rotation around the symmetry axis. Against the huge effect of drag, it must provide a high level of common mode rejection by exploiting in the absence of weight the versatile nature of mechanical suspensions-whose properties can be predicted and measured in the lab-so that they become the solution rather than being the problem. It must be free by design from systematic effects due to differential gas pressure (the radiometer effect) and free from the ubiquitous constraint of narrow gaps (to reduce gas damping noise and electric patch effects) by replacing the capacitance readout with laser interferometry. To check systematics, it must allow multiple measurements, as are made possible by low thermal noise and short integration times.

For all of these aspects, viable solutions exist based on proven technology. The proposed GG experiment aims to test the WEP to $10^{-17}$ at room temperature and incorporates all the required features ([4,14-17]). All future proposals for WEP experiments in space will be confronted with the issues raised by MICROSCOPE and will be compared with the solutions offered by GG.

\section{ACKNOWLEDGMENTS}

Thanks are due to the colleagues of the GG Collaboration for their contributions. The input for this work came during the 656th Wilhelm und Else Heraeus Seminar on "Fundamental Physics in Space" held in Bremen. A. M. N wishes to thank the organizers for their invitation. Special thanks to Manuel Rodrigues, of the MICROSCOPE collaboration, for useful information and discussions. 
[1] R. V. Eötvös, Über die Anziehung der Erde auf verschiedene Substanzen, Mathematische und Naturwissenschaftliche Berichte aus Ungarn, 8, 65 (1890); see also On the gravitation produced by the Earth on different substances, The Abraham Zelmanov Journal 1, 6 (2008).

[2] S. Schlamminger, K.-Y. Choi, T. A. Wagner, J. H. Gundlach, and E. G. Adelberger, Test of the Equivalence Principle Using a Rotating Torsion Balance, Phys. Rev. Lett. 100, 041101 (2008).

[3] T. A. Wagner, S. Schlamminger, J.H. Gundlach, and E. G. Adelberger, Torsion-balance tests of the weak equivalence principle, Classical Quantum Gravity 29, 184002 (2012).

[4] A. M. Nobili and A. Anselmi, Relevance of the weak equivalence principle and experiments to test it: Lessons from the past and improvements expected in space, Phys. Lett. A 382, 2205 (2018).

[5] P. W. Worden, Jr. and C. W. F. Everitt, Test of the Equivalence of Gravitational and Inertial Mass Based on Cryogenic Techniques, in Proc. Int. School of Physics E. Fermi, Course LVI: Experimental Gravitation (Academic Press, New York, 1973), p. 381.

[6] P. W. Worden, Jr., A cryogenic test of the equivalence principle, Ph.D. thesis, Stanford University, Stanford, California, 1976.

[7] P. W. Worden, Jr., Equivalence principle tests in earth orbit, Acta Astronaut. 5, 27 (1978).

[8] E. Fischbach, D. Sudarsky, A. Szafer, C. Talmadge, and S. H. Aronson, Reanalysis of the Eötvös Experiment, Phys. Rev. Lett. 56, 1427 (1986).

[9] J. P. Blaser et al. (including A. M. Nobili), Satellite test of the equivalence principle, Report on the phase A study, ESA/NASA Report No. SCI 93(4) (1993).

[10] P. Touboul, G. Metris, V. Lebat, and A. Robert, The MICROSCOPE experiment, ready for the in-orbit test of the equivalence principle, Classical Quantum Gravity 29, 184010 (2012).

[11] P. Touboul et al., MICROSCOPE Mission: First Results of a Space Test of the Equivalence Principle, Phys. Rev. Lett. 119, 231101 (2017).

[12] V. Josselin, P. Touboul, and R. Kielbasa, Capacitive detection scheme for space accelerometers applications, Sens. Actuators 78, 92 (1999).

[13] P. R. Saulson, Thermal noise in mechanical experiments, Phys. Rev. D 42, 2437 (1990).

[14] R. Pegna, A. M. Nobili, M. Shao, S. G. Turyshev, G. Catastini, A. Anselmi, R. Spero, S. Doravari, G. L. Comandi, and A. De Michele, Abatement of Thermal Noise due to Internal Damping in 2D Oscillators with Rapidly Rotating Test Masses, Phys. Rev. Lett. 107, 200801 (2011).

[15] A. M. Nobili, M. Shao, R. Pegna, G. Zavattini, S. G. Turyshev, D. M. Lucchesi, A. De Michele, S. Doravari, G. L. Comandi, T. R. Saravanan, F. Palmonari, G. Catastini, and A. Anselmi, "Galileo Galilei" (GG): space test of the weak equivalence principle to $10^{-17}$ and laboratory demonstrations, Classical Quantum Gravity 29, 184011 (2012).

[16] "Galileo Galilei” (GG) Website (2018), http://eotvos.dm .unipi.it/.

[17] A. Anselmi and G. Catastini, Design of the GG satellite, Phys. Lett. A 318, 205 (2003).
[18] J. P. Blaser et al., Satellite test of the equivalence principle, Report on the phase A Study, ESA Report No. SCI 96(5) (1996).

[19] M. Rodrigues, MICROSCOPE mission: a test of the equivalence principle in space, 656th WE-Heraeus-Seminar Fundamental Physics in Space, Bremen, 2017, https://www .zarm.uni-bremen.de/fps2017/pdf/Vortraege/RodriguesMicroscope-breme.pdf.

[20] E. Willemenot, Pendule de torsion... suspension électrostatique, Ph.D. thesis, Université Orsay-Paris XI, 1997.

[21] E. Willemenot and P. Touboul, On-ground investigation of space accelerometers noise with an electrostatic torsion pendulum, Rev. Sci. Instrum. 71, 302 (2000).

[22] E. Willemenot and P. Touboul, Electrostatically suspended torsion pendulum, Rev. Sci. Instrum. 71, 310 (2000).

[23] LIGO Facts, Webpage at Caltech, https://www.ligo.caltech .edu/page/facts.

[24] B. Christophe, GRACE and GOCE experience (MICROSCOPE Colloquium II, ONERA, Paris, 2013), http://gram .oca.eu/Ressources_doc/2-Microscope-Colloquium-2013/24 .B.Christophe.GRACE-GOCE.pdf.

[25] P. Touboul, MICROSCOPE mission overview (MICROSCOPE Colloquium II, ONERA, Paris, 2013), http://gram .oca.eu/Ressources_doc/2-Microscope-Colloquium-2013/ 11.P.Touboul.MIssionOverview.pdf.

[26] B. Frommknecht, D. Lamarre, M. Meloni, A. Bigazzi, and R. Floberghagen, GOCE level $1 \mathrm{~b}$ data processing, J. Geod. 85, 759 (2011).

[27] A. Milani, A. M. Nobili, and P. Farinella, Non-gravitational Perturbations and Satellite Geodesy (Adam Hilger Ltd., Bristol 1987).

[28] P. Touboul, Among space fundamental physics missions, MICROSCOPE, a simple free-fall test, Moriond and GPhys Colloquium on gravitational waves and experimental gravity, La Thuile, 2011, http://moriond.in2p3.fr/J11/transparents/ touboul.ppt.

[29] D. Hagedorn et al., MICROSCOPE_fabricating test masses for an in-orbit test of the equivalence principle, Ann. Phys. (Berlin) 525, 720 (2013).

[30] A. M. Nobili, D. Bramanti, G. Comandi, R. Toncelli, E. Polacco, and G. Catastini, Radiometer effect in space missions to test the equivalence principle, Phys. Rev. D Rapid Commun. 63, 101101(R) (2001).

[31] M. Armano et al., Sub-Femto-g Free Fall for Space-Based Gravitational Wave Observatories: LISA Pathfinder Results, Phys. Rev. Lett. 116, 231101 (2016).

[32] M. Armano et al., Beyond the Required LISA Free-Fall Performance: New LISA Pathfinder Results down to $20 \mu \mathrm{Hz}$, Phys. Rev. Lett. 120, 061101 (2018).

[33] A. M. Nobili, D. Bramanti, G. L. Comandi, R. Toncelli, and E. Polacco, Radiometer effect in the Microscope space mission, New Astron. 7, 521 (2002).

[34] A. Cavalleri, G. Ciani, R. Dolesi, A. Heptonstall, M. Hueller, D. Nicolodi, S. Rowan, D. Tombolato, S. Vitale, P. J. Wass, and W. J. Weber, Increased Brownian Force Noise from Molecular Impacts in a Constrained Volume, Phys. Rev. Lett. 103, 140601 (2009).

[35] A. M. Nobili, R. Pegna, M. Shao, S. G. Turyshev, G. Catastini, A. Anselmi, R. Spero, S. Doravari, G. L. Comandi, D. M. Lucchesi, and A. De Michele, Integration 
time in space experiments to test the equivalence principle, Phys. Rev. D 89, 042005 (2014).

[36] M. Armano et al., Capacitive sensing of test mass motion with nanometer precision over millimeter-wide sensing gaps for space-borne gravitational reference sensors, Phys. Rev. D 96, 062004 (2017).

[37] A. M. Nobili, D. Bramanti, E. Polacco, G. Catastini, A. Anselmi, S. Portigliotti, A. Lenti, P. di Giamberardino, S. Monaco, and R. Ronchini, Evaluation of a proposed test of the weak equivalence principle using Earth-orbiting bodies in high-speed co-rotation: Re-establishing the physical bases, Classical Quantum Gravity 16, 1463 (1999).

[38] M. Pisani, G. Mana, and A. M. Nobili, Design of an interferometric displacement sensor with picometer resolution for the Galileo Galilei mission, in 2015 IEEE Metrology for Aerospace, 591-595 (2015), DOI 10.1109/MetroAeroSpace.2015.7180724.

[39] M. Pisani, M. Zucco, and A. M. Nobili, Laboratory tests of a high-precision laser interferometry readout for the
GG experiment in space, in 2016 IEEE Metrology for Aerospace, 260-265 (2016), DOI 10.1109/MetroAeroSpace.2016.7573223.

[40] A. M. Nobili et al., in Major challenges of a high precision test of the equivalence principle in space, in Proceedings of XLVIth Moriond Conference on "Gravitational Waves and Experimental Gravity, edited by E. Augè, J. Dumarchez, and J. Tran Thanh Van (The Gioi Publishers, 2011), p. 341.

[41] A. M. Nobili, GG: Abatement of Thermal Noise by Rotation and Null Checks of an Equivalence Principle Violation Signal to $10^{-17}$, XLVIth Moriond Conference on Gravitational Waves and Experimental Gravity, 2011, http:// moriond.in2p3.fr/J11/transparents/nobili.pdf.

[42] H. Dittus, Bremen Conference on Fundamental Physics in Space, 2017, https://www.zarm.uni-bremen.de/fps2017/.

[43] A. M. Nobili, D. Bramanti, G. L. Comandi, R. Toncelli, E. Polacco, and M. L. Chiofalo, Galileo Galilei-GG: Design, requirements, error budget and significance of the ground prototype, Phys. Lett. A 318, 172 (2003). 